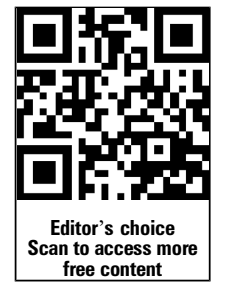

Correspondence to Professor Fiona McQueen, Department of Molecular Medicine and Pathology, University of Auckland, 85 Park Road, Grafton, Auckland 1023, New Zealand; f.mcqueen@auckland.ac.nz

Received 13 May 2013 Revised 20 February 2014 Accepted 21 February 2014 Published Online First 27 March 2014
CrossMark

To cite: McQueen FM. Postgrad Med J

2014;90:332-339.

\title{
MRI in rheumatoid arthritis: a useful tool for the clinician?
}

\author{
Fiona M McQueen
}

\begin{abstract}
Over the last two decades, MRI has emerged as an important clinical tool to assist in the diagnosis and management of rheumatic disease. In rheumatoid arthritis (RA), MRI has improved our understanding of the pathological basis of disease and has provided new information about imaging features that reflect joint inflammation and damage. Using MRI, we can now directly observe inflammation involving the synovial membrane and tenosynovium, plus joint damage including bone erosion and cartilage thinning. Inflammation of bone beneath the joint (osteitis) appears as bone oedema which is a feature unique to MRI and yields important diagnostic and prognostic information in patients with inflammatory arthritis. With the introduction of biologics to rheumatology clinical practice, sensitive tools are required to monitor disease activity and progression, so that the disease suppressing effect of these new agents can be measured. MRI fits the bill for this role as it can inform the clinician about the development of bone erosions well before plain radiography, and its ability to reveal cartilage damage is emerging. The use of MRI as a marker of outcome in clinical trials is being paralleled by its increasing role in the clinic. Both extremity and high field MRI have clinical applications in RA and need to be considered along with other advanced imaging techniques as useful tools to add to the clinician's armamentarium. This review will summarise recent advances in this field and will apply current knowledge to specific clinical scenarios relevant to modern rheumatology practice.
\end{abstract}

\section{INTRODUCTION}

Imaging has always played an important role in rheumatology and has particular significance in the diagnosis and management of rheumatoid arthritis (RA). The detection of radiographic (XR) erosions featured as part of the American College of Rheumatology (ACR) 1987 classification criteria for $\mathrm{RA}^{1}$ and the presence of typical rheumatoid erosions remain indicative of this diagnosis according to the newer ACR/EULAR 2010 criteria, ${ }^{2}$ despite their orientation towards diagnosing patients in the pre-erosive stage. MRI is one of the newer techniques now available for early detection of erosions and it has the added bonus of being capable of imaging pre-erosive and inflammatory joint changes. ${ }^{3}$ Thus, it is increasingly playing an important role to assist the clinician in diagnosing and monitoring disease progression in RA. ${ }^{4}$ While MRI remains relatively expensive, it does have a number of advantages over other forms of imaging. These include its 3D capability, which allows detailed imaging of all joint areas including complex regions such as the wrist and foot which are not fully accessible to ultrasound and where 2D $\mathrm{XR}$ is often inadequate. ${ }^{5}$ MRI images are obtained in a digitised format and can therefore be stored and retrieved in the future for comparison with baseline scans - an advantage also now enjoyed by digitised radiography and ultrasound, using picture archiving and communication system (PACS) systems. There are limitations to MRI related to the intense magnetic field associated, so that scans cannot be performed in patients with pacemakers in situ, where there is a metallic foreign body in the eye or certain types of aneurysm clips in the brain, as these could be dislodged. Some patients cannot tolerate scans because of claustrophobia and scanners cannot accommodate very obese patients. Finally, those with orthopaedic metalware can be scanned but the resulting metal artefact will influence imaging in those regions.

Importantly, MRI is the most inclusive of all modalities when it comes to imaging all relevant tissues as pure radiographic techniques such as $\mathrm{XR}$ and CT cannot reveal the features of joint inflammation such as synovitis and tenosynovitis and no other technique is capable of imaging osteitis; apparent as MRI bone marrow oedema. The MRI features of early and late RA have been very well characterised and studied over the past 15 years and scoring systems have been developed for use in clinical trials to quantify damage and inflammation in the rheumatoid joint. ${ }^{6}$ Finally, MRI scanning has the advantage of not employing ionising radiation and can therefore be used without concern about a cumulative radiation burden. In the following review, the role of MRI scanning in the diagnosis and management of RA will be reviewed with particular emphasis on its relevance in the clinical setting.

\section{BACKGROUND: WHAT INFORMATION CAN WE GET FROM AN MRI SCAN?}

Within the rheumatoid joint, synovitis can be detected on fat-saturated (FS) T1-weighted (T1W) MRI images as thickened synovial membrane (showing its anatomical features), while on T2W or short tau inversion recovery (STIR) imaging, there is high signal within this region, indicating a high water content. This gives a degree of 'functional' information, reflecting the inflammatory process. Regions of synovitis usually enhance on T1W postcontrast images, revealing high vascularity. Similarly, tenosynovitis may be recognised on the same sequences and if signal change occurs within the tendons themselves, this can indicate damage to collagen fibrils, which may sometimes predate tendon rupture. 7 Bone pathology is also extremely well seen using MRI. An erosion appears as a 'step- 
down' break in cortical bone and there are changes in the subcortical bone beneath, where fat is usually replaced by inflammatory tissue (synovium). Thus, loss of fat (which is high signal or 'bright' on non-FS T1W images) from this trabecular bone, and replacement by low signal inflamed synovium, can help reveal the break in the overlying cortex. If the tissue filling the erosion is vascular, postcontrast T1W FS sequences will have high signal and this informs the rheumatologist about disease activity at this particular site. ${ }^{8}$ MRI scanning is the only modality to indicate whether there is inflammation (osteitis) in the bone underlying the joint. This is revealed as bone oedema, which has been shown in RA to represent a region of lymphoplasmacytic inflammation within the bone. ${ }^{9}$ This is often found adjacent to erosions but may also predate the development of erosions and therefore has prognostic significance. ${ }^{10-12}$ Finally, MRI is now being used to image cartilage damage as it occurs in large joints such as the knee ${ }^{13}$ and more recently in the small joints of the carpus. ${ }^{14} 15$ Damage is revealed by thinning of the cartilage band overlying bone and this translates to the XR equivalent of joint space narrowing which is a surrogate marker for the same process. At the knee, changes in cartilage composition leading to altered signal characteristics have been studied extensively ${ }^{16}$ and this is an evolving area in the assessment of rheumatoid joint damage.

\section{HOW RELIABLE IS MRI?}

As is the case for any imaging modality, there are pitfalls associated with the use of MRI to answer important clinical questions. First, the quality of the scan will greatly influence the ability to detect pathological change (such as erosion, synovitis or bone oedema). High field $1.5 \mathrm{~T}$ or $3.0 \mathrm{~T}$ scans are now widely used for diagnostic purposes in general medicine and usually have a high signal to noise ratio with good image resolution. ${ }^{17}$ However, a number of factors can influence this, including whether an appropriate extremity surface coil is used, the patient is positioned properly in the scanner (and obviously is not too obese which may preclude scanning altogether) and patient movement. Patients with severe renal failure may be unsuitable to receive intravenous gadolinium-based contrast agents because of concern over precipitating nephrogenic systemic fibrosis (NSF). ${ }^{18}$ However, NSF has not been reported in patients with a creatine clearance $>60 \mathrm{~mL} / \mathrm{min}$ and in the setting of mild renal impairment, macrocyclic gadolinium chelates can be used as these have higher stability than linear chelates. ${ }^{19}$ If the scan is performed without contrast, it is more difficult to accurately detect synovitis and tenosynovitis, especially when low field $0.2 \mathrm{~T}$ extremity MRI (E-MRI) imaging is used. ${ }^{20}$ A study comparing reliability of these systems concluded that E-MRI performs similarly to conventional high-field MRI for the assessment of bone erosions but is inferior with much more variability for synovitis and bone oedema. ${ }^{21}$ Others have confirmed that E-MRI lacks sensitivity for detection of bone oedema. $^{22}$

Error may occur when MRI scans are interpreted and scored for features reflecting joint inflammation and damage, even when this is done by expert radiologists. A thorough knowledge of the anatomy of the region is required including variants of normal such as sites of nutrient foramina in the carpal bones and ligament attachment sites (these can simulate erosions). The significance of regions of high or low signal must be interpreted with regard to possible partial volume artefacts, inhomogeneity of fat suppression and variations in the magnetic field when the extremities are being imaged. ${ }^{23}$ Finally, even when highly trained readers are used, there is considerable inter-reader variation when MRI scans are scored independently, without consensus decisions. In the clinical setting, decisions are generally based on one radiologist's report, so knowledge of potential variability is important. For imaging the rheumatoid wrist and hand, the Outcome Measures in Rheumatoid Arthritis Clinical Trials (OMERACT) MRI imaging group developed the Rheumatoid Arthritis MRI Score (RAMRIS) in $2003^{6}$ and reference atlas in $2005^{24}$ to improve standardisation of MRI outcomes in clinical trials. Work by this group and others have yielded information on intraclass correlation coefficients for inter-reader reliability which range between 0.8 and 0.95 for the assessment of erosions and bone oedema (high to very high reliability) 2526 and 0.6 and 0.9 for synovitis (moderate to high). ${ }^{26-28}$ Table 1 summarises these reliability data. There has been interest in computer-assisted outlining techniques for the assessment of synovial volume (as a surrogate for synovitis) 2729 and quantitation of bone erosion and oedema ${ }^{30} 31$ but these methods are generally too time-consuming for routine clinical use. The assessment of cartilage change at the rheumatoid wrist may also be achieved reliably according to recent reports ${ }^{14} 26$ and the OMERACT group has developed an MRI joint space narrowing score reflecting cartilage loss. ${ }^{32}$

\section{CLINICAL APPLICATIONS FOR MRI IN RA}

The following three clinical scenarios describe situations where MRI scanning of the wrist was helpful in diagnosing and managing patients with RA.

\section{Diagnosis}

\section{Clinical scenario 1}

A 45-year-old Thai woman has a 3-year history of joint pain in the hands and feet. She has multiple tender joints plus non-articular soft tissue tenderness, no swollen joints and a c-reactive protein $(C R P)<1$ (repeated on multiple occasions). Her anti-cyclic citrullinated peptides (CCP) test was high positive on one occasion and is low positive when retested. Her rheumatoid factor (RF) is also weak positive. Radiography of hands and feet is normal. She is seen by two different physicians on separate occasions, one of whom suspects RA and one who has diagnosed a pain syndrome and thinks RA 'very unlikely'. A 3 T contrast-enhanced MRI scan of the wrist was performed as shown in figure 1 and revealed features indicating a high likelihood of active inflammatory arthritis.

MRI scanning (ideally with contrast-enhancement) of the dominant hand and wrist can help to confirm a clinical diagnosis of RA. Florid synovitis, tenosynovitis, bone oedema (osteitis) and bone erosions have been described in a number of RA patient cohorts. $^{10-123334}$ Although all of these features may occur in osteoarthritis, ${ }^{35}$ mechanical derangements of the wrist and asymptomatic controls, ${ }^{36}$ when they are combined together as a 'full house' as above, this can be a strong pointer towards a diagnosis of RA as reviewed recently by Suter et al. ${ }^{37}$ Duer-Jensen et $a l^{38}$ studied 129 patients with undifferentiated inflammatory arthritis and found the optimal model to predict the onset of future RA incorporated positivity for RF, early morning stiffness $>1 \mathrm{~h}$, hand involvement and MRI bone oedema. This model was correct in $82 \%$ of the 27 patients who developed RA after 1 year. ${ }^{38}$ Tamai et al described similar findings in their cohort of 129 patients with undifferentiated arthritis. Of the 22 patients who were anti-cyclic citrullinated 
Table 1 Inter-reader reliability estimated using intraclass correlation coefficients (ICCs) $(95 \% \mathrm{Cls})^{*}$, for high field MRI studies of the wrist where two or more readers assessed scans separately (perfect agreement between readers gives ICC=1)

\begin{tabular}{|c|c|c|c|c|c|c|c|}
\hline $\begin{array}{l}\text { Study } \\
\text { (in chronological order) }\end{array}$ & MRI strength (T) & No. of readers & Synovitis & Tenosynovitis & Bone oedema & Erosion & Cartilage \\
\hline McQueen et $a l^{3}$ & 1.5 & 2 & 0.74 (0.56 to 0.85$)$ & $0.77(0.76$ to 0.87$)$ & 0.84 (0.72 to 0.91$)$ & $0.77(0.60$ to 0.87$)$ & ND \\
\hline Haavardsholm et al ${ }^{25}$ & 1.5 & 4 & 0.69 (0.47 to 0.89$)$ & ND & 0.79 (0.59 to 0.94 & 0.83 (0.66 to 0.94$)$ & ND \\
\hline Bird et $a l^{21}$ & 1.0 & 3 & 0.73 & ND & 0.73 & 0.52 & ND \\
\hline Durez et $a l^{46 *}$ & 1.5 & 2 & 0.88 (0.68 to 0.96$)$ & ND & $0.82(0.55$ to 0.94$)$ & 0.94 (0.83 to 0.98$)$ & ND \\
\hline McQueen et $a^{26}$ & 3.0 & 3 & 0.63 (0.46 to 0.77 ) & ND & $0.80(0.50$ to 0.91$)$ & 0.79 (0.61 to 0.88$)$ & 0.91 (0.86 to 0.94$)$ \\
\hline Ostergaard et $\left.a\right|^{47 *}$ & 1.5 & 2 & 0.81 & ND & 0.68 & 0.90 & ND \\
\hline Haarvardsholm et al ${ }^{62}$ & 1.5 & 4 & ND & $0.73(0.52$ to 0.90$)$ & ND & ND & ND \\
\hline Ostergaard et $a l^{32}$ & 1.0 & 2 & ND & ND & ND & ND & 0.83 (median) \\
\hline
\end{tabular}

*Single measures ICCs given, 95\% Cls where provided.

peptides (CCP) positive in whom MRI bone oedema was present, all progressed to RA at 1 year, with a positive predictive value (PPV) of $100 \% .{ }^{39}$ Narvaez et al examined 40 undifferentiated arthritis patients and predicted a later diagnosis of RA on the basis of MRI synovitis with either erosions and/or bone oedema. The MRI scan findings correctly predicted the onset of RA in 31 of 33 patients, giving a sensitivity of $100 \%$ and specificity of $78 \%$, contrasting with anti-CCP positivity which gave a sensitivity of $23 \%$ and specificity of $100 \%{ }^{40}$ Interestingly, the two patients with 'false positive' MRI scans later developed PsA and antisynthetase syndrome, respectively. ${ }^{40}$ Much interest is now focusing on the pre-RA state where patients are anti-CCP antibody positive and may have arthralgia but not yet true arthritis. Krabben et al recently reported $1.5 \mathrm{~T}$ MRI imaging of the hands and feet in 21 ACPA-positive arthralgia patients and found a correlation between the MRI inflammation score and anti-CCP positivity ${ }^{41}$ suggesting a pathological link between these parameters and their importance in disease initiation. ${ }^{42}$ In summary, MRI scanning can be most helpful in determining a diagnosis of RA, especially when there is difficulty discriminating between arthralgia in a pain sensitive patient and true inflammatory arthritis.

\section{Prognosis}

\section{Clinical scenario 2}

A 60-year-old woman of Caucasian ethnicity was diagnosed with RA 2 years ago and was treated initially with 3 months of methotrexate at $20 \mathrm{mg} /$ week to which she did not respond adequately, followed by triple therapy with methotrexate, sulfasalazine and hydroxychloroquine at standard doses. On assessment, the patient reported 'feeling fine' and clinical examination revealed two tender and four swollen joints. The CRP was $27 \mathrm{mg} / \mathrm{L}(0-5)$ and the DAS28 CRP=3.51 (moderate disease activity $^{43}$ ). Her XRs showed degenerative change only. An MRI scan was performed to investigate prognosis and likelihood to progress to radiographic erosion/joint damage. This showed widespread bone oedema and synovitis with small erosions (figure 2A). Management was changed to combination methotrexate-leflunomide.

MRI can be a very helpful addition to clinical judgement when trying to determine how likely a patient is to develop long-term joint damage. In a study published in 1999, McQueen et al studied 42 patients with recent-onset RA, and found that a high combined score for MRI synovitis, tenosynovitis and bone oedema was strongly predictive of the development of erosions after 1 year (PPV 93\%). ${ }^{44}$ Similarly, a low score strongly predicted a lack of erosions after 1 year (NPV 0.91). This study predated the use of biological disease modifying antirheumatic drugs (bDMARDs) but $70 \%$ were receiving conventional DMARDs (cDMARDs) of the time including 25\% on methotrexate. The same patients were then followed over 6 years and MRI prognostic markers explored. ${ }^{3} 1044$ At individual sites within the wrist, bone oedema was followed by MRI erosion after 1 year with an OR of $6.5 .{ }^{44}$ After 6 years, paired observations were available at 407 sites in 31 patients and baseline MRI bone oedema was strongly associated with 6 year XR joint erosion and joint space narrowing. ${ }^{10}$ Haavardsholm et $a l^{11}$ confirmed bone oedema to be the most important independent predictor for radiographic progression with a baseline RAMRIS score of 2 increasing the risk of progression threefold, and contributing more than the other MRI findings (synovitis, tenosynovitis, erosions) and conventional measures (28-SJC, 28-TJC, ESR, c-reactive protein (CRP), DAS28, anti-CCP). Hetland et al described a similar result in a larger Danish cohort at 2 years ${ }^{45}$ and 5 years. ${ }^{12}$ These patients were enrolled into the CIMESTRA study; a double-blind, placebo-controlled trial in patients with early active RA, treated aggressively with traditional DMARDs plus or minus cyclosporine. ${ }^{12}$ In the 130 who had a baseline MRI scan of the hand, the RAMRIS bone oedema score $^{6}$ was the only independent predictor of erosive progression at 2 years, and alone explained a very large $41 \%$ of the variance $(\mathrm{p}<0.001)$, while the anti-CCP status was much less predictive of outcome $(p=0.08) .^{45}$

Thus, in situations where the clinician is trying to decide whether a patient is adequately controlled on current therapy, especially where there is discordance between reports of pain and inflammatory disease activity scores, MRI of the wrist may be very helpful.

\section{Monitoring disease activity and remission}

\section{Clinical scenario 3}

Management was changed for the patient in scenario 2 from triple therapy to combination methotrexate ( $20 \mathrm{mg} / \mathrm{week})$ and leflunomide $10 \mathrm{mg} / \mathrm{d}$. She did well on these medications over the next 2 years apart from developing low grade leucopenia which prevented escalation of the leflunomide dose to $20 \mathrm{mg} / \mathrm{d}$. A further MRI scan was performed to monitor her response to therapy and revealed a marked reduction in synovitis and bone oedema (figure 2B). The same therapy was therefore continued. 

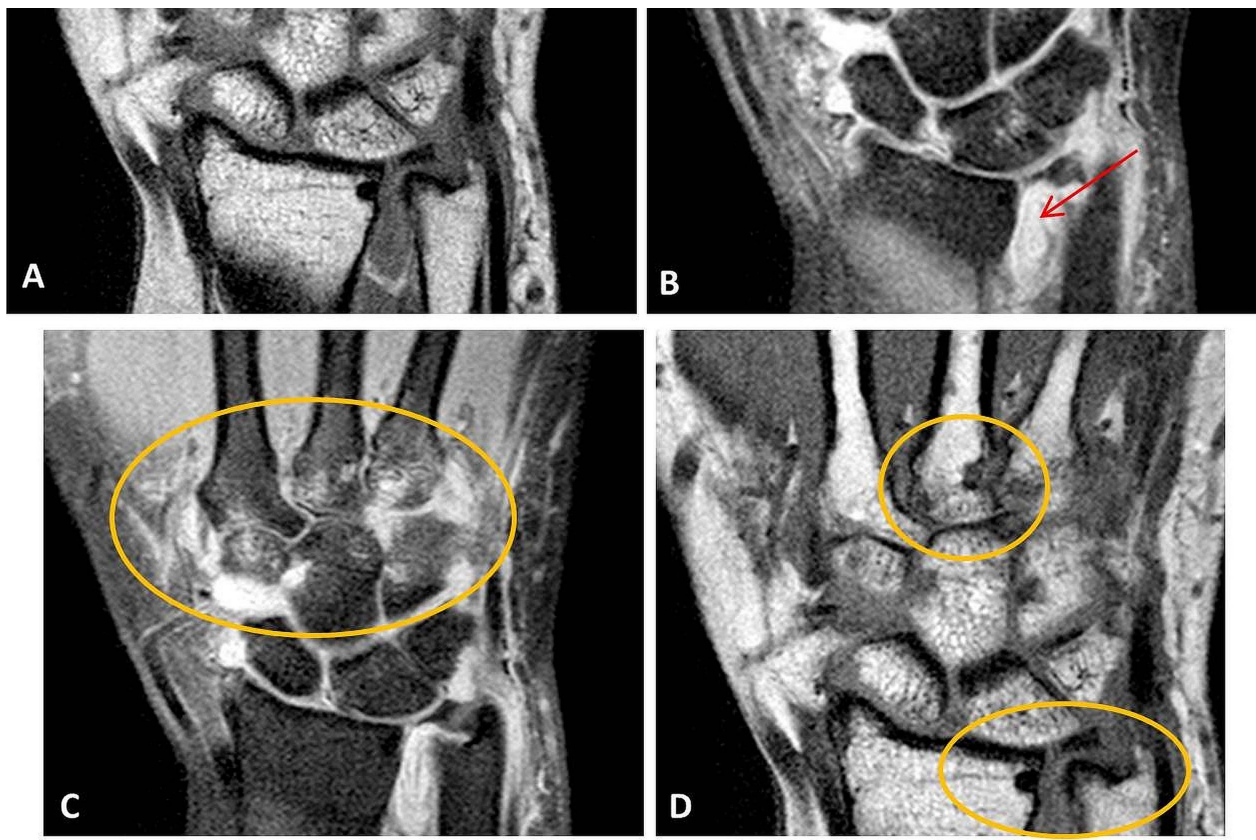

Figure 1 Scenario 1. (A) Coronal T1W image of the wrist showing synovial hypertrophy within the distal radioulnar joint (B) coronal fat suppressed (FS) T1w post-contrast image shows enhancement of this region postcontrast (arrow) indicating the presence of active synovitis and effusion. (C) Proton density coronal image revealing extensive bone oedema (osteitis) throughout 2nd, 3rd and 4th metacarpal bases as well as trapezoid and distal hamate (circle). (D) T1W coronal image showing erosions at the base of the 3rd metacarpal and at the distal radius and ulna (circles).

MRI can be used as a sensitive measure of response to therapy as described by Haavardsholm et al who studied 36 RA patients at initiation of antitumour necrosis factor (anti-TNF) therapy and over the following 12 months. After 3 months, there was a marked reduction in all measures of inflammation (clinical, ultrasound and MRI parameters). ${ }^{33}$ The most responsive was a composite of MRI synovitis, tenosynovitis and bone oedema (osteitis), which had more than double the sensitivity to change over time of the next non-MRI measure which was the DAS28. These authors concluded that the MRI inflammation score is a promising outcome measure for use in clinical practice. A number of clinical trials have employed MRI measures to assess drug efficacy, as results are available long before changes appear on plain XR. Durez et al reported that methotrexate/infliximab was superior to methotrexate alone for reducing MRI synovitis and bone oedema in early RA. Progression of MRI erosions was also greater in the group not receiving anti-TNF therapy. ${ }^{46}$ Imaging results from the GO-BEFORE study trialling golimu$\mathrm{mab}$ in RA have now been reported ${ }^{47}$ and showed that both synovitis and osteitis (bone oedema) fell post-anti-TNF therapy.

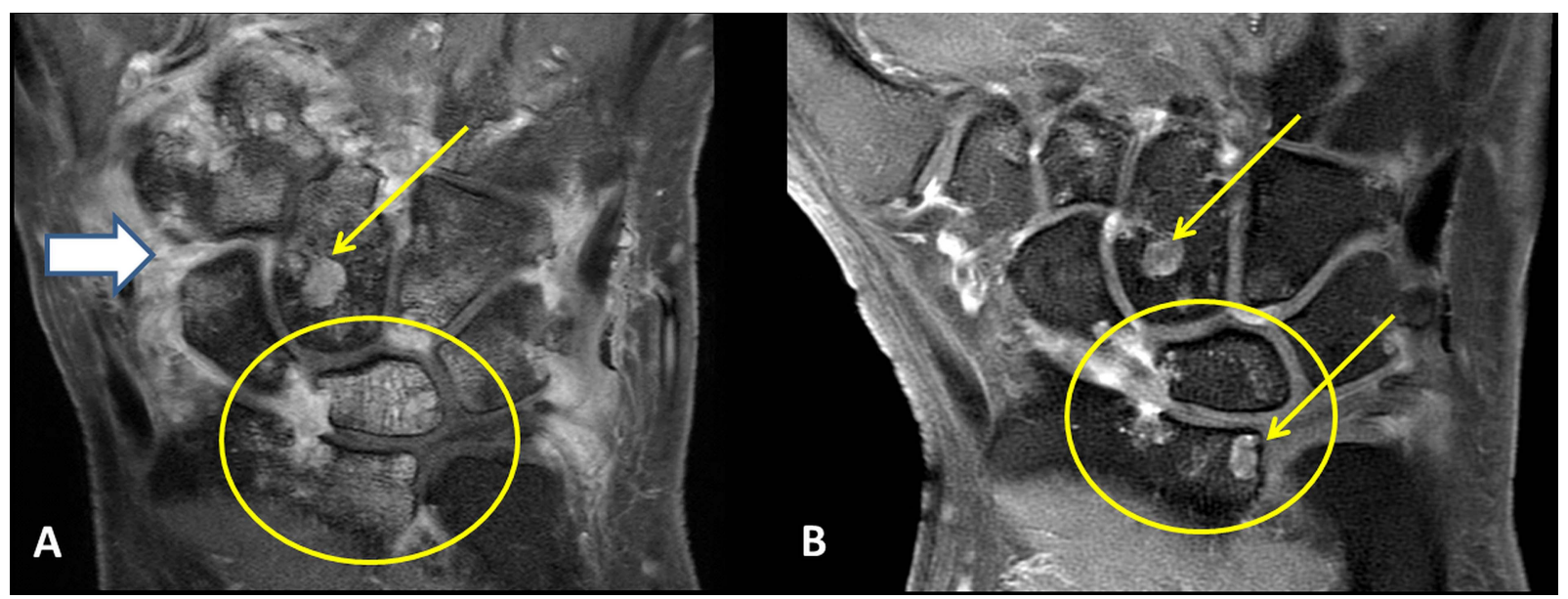

Figure 2 (A) Scenario 2: MRI wrist scan taken from rheumatoid arthritis patient in 2009. Coronal FS T1W postcontrast image of the wrist showing active synovitis at the mid-carpal joint with enhancing synovium adjacent to the scaphoid (wide arrow), an erosion within the capitate (thin arrow) and extensive bone oedema within the lunate and opposite region of the distal radius (circle). (B) Scenario 3: Matching scan taken 3 years later after management with combination methotrexate and leflunomide shows persistence of the erosion within the capitate (arrow) and a new erosion within the distal radius at the site of previous bone oedema (arrow), but no bone oedema within the lunate (circle) and much less synovitis at the carpal joints. 
At 52 weeks, XR progression was associated with greater baseline values for synovitis and osteitis and less early improvement in these parameters at 3 months. ${ }^{28}$ Very recently, the Danish OPERA study reported MRI inflammation responses in early RA patients receiving methotrexate/intra-articular glucocorticoid injections, with or without additional adalimumab. ${ }^{49}$ A marked fall in MRI synovitis, osteitis and tenosynovitis was detected in both groups. Interestingly, osteitis scores decreased significantly during the 12-month follow-up in the adalimumab group, but not in the placebo group, suggesting that this could prefigure the reduced erosion progression known to be associated with anti-TNF therapy. These data provide a rationale for using MRI scanning as a sensitive means to detect response to therapy. However, cost and practical difficulties associated with obtaining scans preclude this strategy in many patients, especially where a clinical response is clearly apparent.

\section{IS THE PATIENT IN REMISSION?}

Both MRI and ultrasound studies have revealed a major discrepancy between clinical and imaging features of disease remission in RA. In 1996, Brown et al reported that in asymptomatic RA patients with normal joints, 96.2\% demonstrated synovitis and $46.4 \%$ bone oedema on MRI, while synovial hypertrophy was identified by grey-scale ultrasound in $73.3 \%$ of patients, with associated power Doppler flow on power Doppler ultrasound (PDUS) in $43.3 \% .{ }^{50}$ Others have since reported similar findings in larger cohorts using $\mathrm{MRI}^{51}$ and ultrasound. ${ }^{52}$ This no doubt goes some way towards explaining why progression of joint damage has been observed in patients in apparent clinical remis$\operatorname{sion}^{53}$ and has led to a discussion about whether MRI and ultrasound outcomes should be incorporated into definitions of imaging remission. ${ }^{54}$ In a recent study by the OMERACT MRI working group, MRI data from several RA cohorts were combined and analysed for evidence of a 'safe level' of joint inflammation, below which erosion progression does not occur. ${ }^{55} \mathrm{~A}$ RAMRIS synovitis score of 5 or lower was found to fit these criteria but bone oedema was relatively rare in this dataset and further studies using uniform and sensitive MRI protocols are still required. For the clinician, the disparity between clinical and imaging evidence of disease activity leads to a tension between the desirability of achieving complete disease control (as revealed by sensitive imaging techniques) and the avoidance of toxicity from multiple bDMARD and non-bDMARD therapies. This area will continue to be a challenging one for clinicians and their patients.

\section{HOW ARE SYNOVITIS AND OSTEITIS RELATED?}

Both synovitis and osteitis are vascular lesions, the latter revealed as such by Hodgson et al who found postcontrast-enhancement of bone oedema regions to match signal intensity on $\mathrm{T} 2 \mathrm{~W}$ images. ${ }^{56}$ Our own imaging/histopathological studies indicated a significant 'floor effect' for T2W bone oedema so that mild degrees of osteitis from resected periarticular bone were not detectable on matching MRI scans. However, more intense osteitis appeared as regions of bone oedema, with increasing grades matching each other above a threshold level. ${ }^{57} \mathrm{~T} 2 \mathrm{~W}$ images may also be relatively insensitive to minor changes in osteitis as the relative enhancement ratio of bone marrow in the study by Hodgson and colleagues fell significantly after 2 weeks of anti-TNF $\alpha$ treatment, while no effect was seen on the RAMRIS bone oedema score. ${ }^{56}$ Much remains unknown about how synovitis and bone oedema (osteitis) impact upon one another in RA and critically whether they occur as separate pathological processes operating in parallel or are linked causally. ${ }^{42}$ Very few studies have performed MRI and ultrasound systematically over time to determine how often osteitis and/or synovitis resolve completely in treated RA and most available data are currently owned by Pharma companies with commercial interests in how they are presented. Thus, there is a need for the inclusion of MRI and ultrasound outcome measures in investigator-initiated treat-to-target studies of responses to cDMARDs and bDMARDs in RA.

\section{Comparison with other imaging modalities}

Many of the important new developments in MRI scanning have been paralleled by similar findings using ultrasound. Several ultrasound studies have confirmed the importance of subclinical disease activity with increased synovial thickness on grey-scale ultrasound and positive scores for synovial vascularity on PDUS in RA patients who are in apparent clinical remission. ${ }^{58} 59$ Naredo et $a l^{60}$ showed that a fall in PDUS signal in clinically active joints predicted a lack of damage progression in those joints over time. Although bone oedema cannot be detected by ultrasound, there is an intriguing association between PDUS signal in the synovium above the joint and bone oedema in the bone below, which were highly correlated in a Danish study. ${ }^{61}$ There are strengths and weaknesses of both forms of imaging. MRI has the benefits of offering high image resolution and is capable of detecting all the important aspects. of joint inflammation and damage, including bone oedema and cartilage thinning, at all joint sites, especially the highly informative region of the wrist. Downsides of MRI include prolonged scanning time (especially when contrast is given), potential in some patients for claustrophobia and high cost. Ultrasound is user-friendly and low cost but not all joints are accessible (especially the wrist), reproducibility is variable ${ }^{34}$ and bone oedema cannot be detected. Other imaging modalities such as XR and CT have important roles in monitoring damage progression in RA but are not capable of depicting inflammation.

\section{SUMMARY AND CONCLUSIONS}

To summarise, MRI scanning is a major new tool for the rheumatologist who is investigating and managing patients with RA. It is extremely sensitive for detecting erosions and also provides information about other aspects of joint damage such as cartilage change and tendon rupture. MRI indicators of inflammation include synovitis, tenosynovitis and osteitis and these can separately and collectively be used as indicators of disease activity. There is strong evidence from multiple centres that detection of osteitis is important both to assist in making the diagnosis of RA in those with undifferentiated arthritis and to predict erosive progression in those with established disease. MRI parameters of inflammation are now being used in clinical trials of biological agents to indicate early efficacy and could be employed by the clinician similarly to guide patient management. Complete imaging remission may be unobtainable in many patients, given the evidence that low grade MRI synovitis and osteitis are common even in clinical remission. However, the 'treat to target' philosophy would dictate that imaging remission is the ultimate goal of RA management, which aims to prevent ongoing joint damage. The challenge for the rheumatologist will be to achieve this for his/her patients without adverse effects associated with drug toxicity. 
Main messages

- MRI inflammation scores for synovitis, tenosynovitis and bone oedema can be used to monitor response to therapy in rheumatoid arthritis (RA).

- MRI bone oedema (osteitis) is predictive of erosive progression in RA patients.

- MRI erosions and cartilage narrowing are indicators of damage progression in RA.

\section{Current research questions}

- Does MRI bone oedema (osteitis) predict the progression of cartilage damage in RA?

- Should MRI scans of the wrist be obtained at presentation in RA patients and will this improve management?

- Is imaging remission necessary to prevent damage in RA joints?

\section{Key references}

- McQueen FM, Benton N, Perry D, et al. Bone edema scored on magnetic resonance imaging scans of the dominant carpus at presentation predicts radiographic joint damage of the hands and feet six years later in patients with rheumatoid arthritis. Arthritis Rheum 2003;48:1814-27.

- Brown AK, Quinn MA, Karim Z, et al. Presence of significant synovitis in rheumatoid arthritis patients with disease-modifying antirheumatic drug-induced clinical remission: evidence from an imaging study may explain structural progression. Arthritis Rheum 2006;54:3761-73.

- Hetland ML, Stengaard-Pedersen K, Junker P, et al. Radiographic progression and remission rates in early rheumatoid arthritis-MRI bone oedema and anti-CCP predicted radiographic progression in the 5-year extension of the double-blind randomised CIMESTRA trial. Ann Rheum Dis 2010;69:1789-95.

- Peterfy CG, DiCarlo JC, Olech E, et al. Evaluating joint-space narrowing and cartilage loss in rheumatoid arthritis by using MRI. Arthritis Res Ther 2012;14:R131.

- Ostergaard M, Emery P, Conaghan PG, et al. Significant improvement in synovitis, osteitis, and bone erosion following golimumab and methotrexate combination therapy as compared with methotrexate alone: a magnetic resonance imaging study of 318 methotrexate-naive rheumatoid arthritis patients. Arthritis Rheum 2011;63:3712-22.

\section{Self assessment questions}

Answer true (T) or false (F) for the below,

1. The finding of MRI bone oedema in RA joints is associated with the following (true/false)

A. A lymphoplasmacytic infiltrate in subchondral bone.

B. Erosion progression in the same region in many cases.

C. Synovitis.

D. HLA-DRB $1{ }^{*} 0401$.

E. Female sex.

2. Which of the following is true/false regarding MRI synovitis in RA?

A. Synovitis can be detected most accurately using postcontrast T1W MRI scans.

B. Is observed in less than $50 \%$ of patients with active disease.

C. Is common in patients in clinical remission.

D. Is commonly associated with tenosynovitis and osteitis.

E. Is reduced in patients responding to anti-TNF therapy.

3. Which of the following is true/false regarding erosions in RA as detected by imaging?

A. MRI erosions are often detectable 6-12 months before they are apparent on X-ray.

B. MRI is several times more sensitive than $\mathrm{X}$-ray for detection of erosions.

C. MRI can detect erosions at sites that are not imaged adequately by ultrasound.

D. CT scanning is slightly more specific for identifying erosions than MRI.

E. Regions of MRI bone oedema invariably progress to erosions.

4. Undifferentiated arthritis is more likely to progress to RA in patients who have (true/false)

A. Ultrasound evidence of synovitis at the carpus.

B. MRI bone oedema at the carpus.

C. MRI synovitis.

D. MRI tenosynovitis.

E. Rupture of the triangular fibrocartilage.

5. The following features may be found in RA patients who have no swollen or tender joints (true/false)
A. MRI synovitis.
B. MRI bone oedema (osteitis).
C. MRI tenosynovitis.
D. MRI erosions.
E. MRI evidence of cartilage space narrowing.

Acknowledgements The author wishes to acknowledge the assistance of MRI radiographers/technologists affiliated with the Specialist Radiology \& MRI Greenlane group for assistance with images, and other members of the Auckland

Rheumatology Imaging group in this field of musculoskeletal imaging research.

Competing interests None.

Provenance and peer review Not commissioned; externally peer reviewed. 


\section{REFERENCES}

1 Arnett FC, Edworthy SM, Bloch DA, et al. The American Rheumatism Association 1987 revised criteria for the classification of rheumatoid arthritis. Arthritis Rheum 1988;31:315-24.

2 Aletaha D, Neogi T, Silman AJ, et al. 2010 Rheumatoid arthritis classification criteria: an American College of Rheumatology/European League Against Rheumatism collaborative initiative. Arthritis Rheum 2010;62:2569-81.

3 McQueen FM, Stewart N, Crabbe J, et al. Magnetic resonance imaging of the wrist in early rheumatoid arthritis reveals a high prevalence of erosions at four months after symptom onset. Ann Rheum Dis 1998;57:350-6.

4 Troum OM, Pimienta O, Olech E. Magnetic resonance imaging applications in early rheumatoid arthritis diagnosis and management. Rheum Dis Clin North Am 2012;38:277-97

5 McQueen FM. Magnetic resonance imaging in early inflammatory arthritis: what is its role? Rheumatology 2000;39:700-6.

6 Østergaard M, Peterfy C, Conaghan $\mathrm{P}$, et al. OMERACT rheumatoid arthritis magnetic resonance imaging studies. Core set of MRI acquisitions, joint pathology definitions, and the OMERACT RA-MRI scoring system. J Rheumatol 2003;30:1385-6.

7 McQueen F, Beckley V, Crabbe J, et al. Magnetic resonance imaging evidence of tendinopathy in early rheumatoid arthritis predicts tendon rupture at six years. Arthritis Rheum 2005;52:744-51.

8 Jevtic V, Watt I, Rozman B, et al. Prognostic value of contrast enhanced Gd-DTPA MRI for development of bone erosive changes in rheumatoid arthritis. $\mathrm{Br} \mathrm{J}$ Rheumatol 1996:35(Suppl 3):26-30.

9 Dalbeth N, Smith T, Gray S, et al. Cellular characterisation of magnetic resonance imaging bone oedema in rheumatoid arthritis; implications for pathogenesis of erosive disease. Ann Rheum Dis 2009;68:279-82.

10 McQueen FM, Benton N, Perry D, et al. Bone edema scored on magnetic resonance imaging scans of the dominant carpus at presentation predicts radiographic joint damage of the hands and feet six years later in patients with rheumatoid arthritis. Arthritis Rheum 2003:48:1814-27.

11 Haavardsholm EA, Boyesen $\mathrm{P}$, Ostergaard $\mathrm{M}$, et al. Magnetic resonance imaging findings in 84 patients with early rheumatoid arthritis: bone marrow oedema predicts erosive progression. Ann Rheum Dis 2008;67:794-800.

12 Hetland ML, Stengaard-Pedersen K, Junker $\mathrm{P}$, et al. Radiographic progression and remission rates in early rheumatoid arthritis-MRI bone oedema and anti-CCP predicted radiographic progression in the 5-year extension of the double-blind randomised CIMESTRA trial. Ann Rheum Dis 2010;69:1789-95.

13 Gandy SJ, Brett AD, Dieppe PA, et al. Measurement of cartilage volumes in rheumatoid arthritis using MRI. Br J Radiol 2005;78:39-45.

14 Peterfy CG, DiCarlo JC, Olech E, et al. Evaluating joint-space narrowing and cartilage loss in rheumatoid arthritis by using MRI. Arthritis Res Ther 2012; $14:$ R131.

15 McQueen FM, McHaffie A, Clarke A, et al. MRI osteitis predicts cartilage damage at the wrist in RA: a three year prospective $3 T$ - MRI study examining cartilage damage. Arthritis Res Ther 2014;16:R33.

16 De Maeseneer M, Shahabpour M, Van Roy P, et al. MRI of cartilage and subchondral bone injury. A pictorial review. JBR-BTR 2008;91:6-13.

17 Peterfy C. Structural characterization of rheumatoid arthritis by MRI: applications in clinical research and in clinical practice. J Musculoskeletal Neuronal Interact 2004:4:360-1.

18 Cowper SE, Kuo PH, Bucala R. Nephrogenic systemic fibrosis and gadolinium exposure: association and lessons for idiopathic fibrosing disorders.[comment]. Arthritis Rheum 2007;56:3173-5

19 Morcos SK. Nephrogenic systemic fibrosis following the administration of extracellular gadolinium based contrast agents: is the stability of the contrast agent molecule an important factor in the pathogenesis of this condition? [Erratum appears in Br J Radiol. 2007 Jul;80(955):586]. Br J Radiol 2007;80: 73-6.

20 Ostergaard M, Conaghan PG, O'Connor $\mathrm{P}$, et al. Reducing invasiveness, duration, and cost of magnetic resonance imaging in rheumatoid arthritis by omitting intravenous contrast injection-Does it change the assessment of inflammatory and destructive joint changes by the OMERACT RAMRIS? J Rheumatol 2009:36:1806-10.

21 Bird P, Ejbjerg B, Lassere $M$, et al. A multireader reliability study comparing conventional high-field magnetic resonance imaging with extremity low-field MRI in rheumatoid arthritis. J Rheumatol 2007;34:854-6.

22 Ejbjerg BJ, Narvestad E, Jacobsen $\mathrm{S}$, et al. Optimised, low cost, low field dedicated extremity MRI is highly specific and sensitive for synovitis and bone erosions in rheumatoid arthritis wrist and finger joints: comparison with conventional high field MRI and radiography. Ann Rheum Dis 2005;64:1280-7.

23 McQueen F, Ostergaard M, Peterfy C, et al. Pitfalls in scoring MR images of rheumatoid arthritis wrist and metacarpophalangeal joints. Ann Rheum Dis 2005;64 (Suppl 1):i48-55.

24 Østergaard M, Edmonds J, McQueen F, et al. An introduction to the EULAR-OMERACT rheumatoid arthritis MRI reference image atlas. Ann Rheum Dis 2005;64(Suppl 1):i3-7.
25 Haavardsholm EA, Ostergaard M, Ejbjerg BJ, et al. Reliability and sensitivity to change of the OMERACT rheumatoid arthritis magnetic resonance imaging score in a multireader, longitudinal setting. Arthritis Rheum 2005;52:3860-7.

26 McQueen FM, Clarke A, McHaffie A, et al. Assessment of cartilage loss at the wrist in rheumatoid arthritis using a new MRI scoring system. Ann Rheum Dis 2010;69:1971-5

27 Chand AS, McHaffie A, Clarke AW, et al. Quantifying synovitis in rheumatoid arthritis using computer-assisted manual segmentation with 3 Tesla MRI scanning. J Magn Reson Imaging 2011;33:1106-13.

28 Conaghan $\mathrm{P}$, Lassere $\mathrm{M}$, Ostergaard $\mathrm{M}$, et al. OMERACT rheumatoid arthritis magnetic resonance imaging studies. Exercise 4: an international multicenter longitudinal study using the RA-MRI Score. J Rheumatol 2003;30:1376-9.

29 Østergaard M, Stoltenberg M, Lovgreen-Nielsen $\mathrm{P}$, et al. Magnetic resonance imaging-determined synovial membrane and joint effusion volumes in rheumatoid arthritis and osteoarthritis: comparison with the macroscopic and microscopic appearance of the synovium.[see comment]. Arthritis Rheum 1997;40:1856-67.

30 Crowley AR, Dong J, McHaffie A, et al. Measuring bone erosion and edema in rheumatoid arthritis: a comparison of manual segmentation and RAMRIS methods. J Magn Reson Imaging 2011;33:364-71.

31 Bird $\mathrm{P}$, Joshua $\mathrm{F}$, Lassere $M$, et al. Training and calibration improve inter-reader reliability of joint damage assessment using magnetic resonance image scoring and computerized erosion volume measurement. J Rheumatol 2005;32:1452-8.

32 Ostergaard M, Boyesen $\mathrm{P}$, Eshed I, et al. Development and preliminary validation of a magnetic resonance imaging joint space narrowing score for use in rheumatoid arthritis: potential adjunct to the OMERACT RA MRI scoring system. J Rheumatol 2011;38:2045-50.

33 Haavardsholm EA, Ostergaard M, Hammer HB, et al. Monitoring anti-TNFalpha treatment in rheumatoid arthritis: responsiveness of magnetic resonance imaging and ultrasonography of the dominant wrist joint compared with conventional measures of disease activity and structural damage. Ann Rheum Dis 2009;68:1572-9.

34 Boyesen $P$, Haavardsholm EA, van der Heijde D, et al. Prediction of MRI erosive progression: a comparison of modern imaging modalities in early rheumatoid arthritis patients. Ann Rheum Dis 2011;70:176-9.

35 Conaghan P. Is MRI useful in osteoarthritis? Best Pract Res Clin Rheumatol 2006;20:57-68

36 Iordache SD, Rowan R, Garvin GJ, et al. Prevalence of triangular fibrocartilage complex abnormalities on MRI scans of asymptomatic wrists. J Hand Surg Am 2012;37:98-103.

37 Suter LG, Fraenkel L, Braithwaite RS. Role of magnetic resonance imaging in the diagnosis and prognosis of rheumatoid arthritis. Arthritis Care Res 2011;63:675-88.

38 Duer-Jensen A, Horslev-Petersen $\mathrm{K}$, Hetland ML, et al. Bone edema on magnetic resonance imaging is an independent predictor of rheumatoid arthritis development in patients with early undifferentiated arthritis. Arthritis Rheum 2011;63:2192-202.

39 Tamai M, Kawakami A, Uetani M, et al. A prediction rule for disease outcome in patients with undifferentiated arthritis using magnetic resonance imaging of the wrists and finger joints and serologic autoantibodies. Arthritis Rheum 2009:61:772-8

40 Narvaez J, Sirvent E, Narvaez JA, et al. Usefulness of magnetic resonance imaging of the hand versus anticyclic citrullinated peptide antibody testing to confirm the diagnosis of clinically suspected early rheumatoid arthritis in the absence of rheumatoid factor and radiographic erosions. Semin Arthritis Rheum 2008:38:101-9.

41 Krabben A, Stomp W, van der Heijde DMFM, et al. MRI of hand and foot joints of patients with anticitrullinated peptide antibody positive arthralgia without clinical arthritis. Ann Rheum Dis 2013;72:1540-4.

42 McQueen FM. Bone marrow edema and osteitis in rheumatoid arthritis: the imaging perspective. Arthritis Res Ther 2012;14:224.

43 Prevoo ML, van't Hof MA, Kuper HH, et al. Modified disease activity scores that include twenty-eight-joint counts. Development and validation in a prospective longitudinal study of patients with rheumatoid arthritis. Arthritis Rheum 1995:38:44-8.

44 McQueen FM, Stewart N, Crabbe J, et al. Magnetic resonance imaging of the wrist in early rheumatoid arthritis reveals progression of erosions despite clinical improvement. Ann Rheum Dis 1999;58:156-63.

45 Hetland ML, Ejbjerg B, Horslev-Petersen K, et al. MRI bone oedema is the strongest predictor of subsequent radiographic progression in early rheumatoid arthritis. Results from a 2-year randomised controlled trial (CIMESTRA). Ann Rheum Dis 2009;68:384-90

46 Durez P, Malghem J, Nzeusseu Toukap A, et al. Treatment of early rheumatoid arthritis: a randomized magnetic resonance imaging study comparing the effects of methotrexate alone, methotrexate in combination with infliximab, and methotrexate in combination with intravenous pulse methylprednisolone. Arthritis Rheum 2007;56:3919-27.

47 Ostergaard M, Emery P, Conaghan PG, et al. Significant improvement in synovitis, osteitis, and bone erosion following golimumab and methotrexate combination therapy as compared with methotrexate alone: a magnetic resonance imaging study of 318 methotrexate-naive rheumatoid arthritis patients. Arthritis Rheum 2011:63:3712-22. 
48 Baker JF, Østergaard M, Emery $\mathrm{P}$, et al. Early MRI measures independently predict 1-year and 2-year radiographic progression in rheumatoid arthritis: secondary analysis from a large clinical trial. Ann Rheum Dis. Published Online First: 31 July 2013. doi:10.1136/annrheumdis-2013-203444.

49 Axelsen MB, Eshed I, Hørslev-Petersen K, et al. A treat-to-target strategy with methotrexate and intra-articular triamcinolone with or without adalimumab effectively reduces MRI synovitis, osteitis and tenosynovitis and halts structural damage progression in early rheumatoid arthritis: results from the OPERA randomised controlled trial. Ann Rheum Dis. Published Online First: 16 Jan 2014 doi:10.1136/annrheumdis-2013-204537

50 Brown AK, Quinn MA, Karim Z, et al. Presence of significant synovitis in rheumatoid arthritis patients with disease-modifying antirheumatic drug-induced clinical remission: evidence from an imaging study may explain structural progression. Arthritis Rheum 2006:54:3761-73.

51 Gandjbakhch F, Conaghan PG, Ejbjerg B, et al. Synovitis and osteitis are very frequent in rheumatoid arthritis clinical remission: results from an MRI study of 294 patients in clinical remission or low disease activity state. J Rheumatol 2011;38:2039-44.

52 Scire CA, Montecucco C, Codullo V, et al. Ultrasonographic evaluation of joint involvement in early rheumatoid arthritis in clinical remission: power Doppler signal predicts short-term relapse. Rheumatology 2009;48:1092-7.

53 Molenaar ET, Voskuyl AE, Dinant HJ, et al. Progression of radiologic damage in patients with rheumatoid arthritis in clinical remission. Arthritis Rheum 2004;50:36-42.

54 Haavardsholm EA, Lie E, Lillegraven S. Should modern imaging be part of remission criteria in rheumatoid arthritis? Best Prac Res Clin Rheumatol 2012;26:767-85.

55 Gandjbakhch F, Haavardsholm EA, Conaghan PG, et al. Determining a magnetic resonance imaging inflammatory activity acceptable state without subsequent radiographic progression in rheumatoid arthritis: results from a followup MRI study of 254 patients in clinical remission or low disease activity. J Rheumatol 2014;41:398-406.

56 Hodgson R, Grainger A, O'Connor P, et al. Dynamic contrast enhanced MRI of bone marrow oedema in rheumatoid arthritis. Ann Rheum Dis 2008;67:270-2.
57 McQueen FM, Gao A, Østergaard M, et al. High grade MRI bone oedema is common within the surgical field in rheumatoid arthritis patients undergoing joint replacement and is associated with osteitis in subchondral bone. Ann Rheum Dis 2007:66:1581-7.

58 Saleem B, Brown AK, Keen $\mathrm{H}$, et al. Should imaging be a component of rheumatoid arthritis remission criteria? A comparison between traditional and modified composite remission scores and imaging assessments. Ann Rheum Dis 2011;70:792-8.

59 Sakellariou G, Scire CA, Verstappen SMM, et al. In patients with early rheumatoid arthritis, the new ACR/EULAR definition of remission identifies patients with persistent absence of functional disability and suppression of ultrasonographic synovitis. Ann Rheum Dis 2013:72:245-9.

60 Naredo E, Collado P, Cruz A, et al. Longitudinal power Doppler ultrasonographic assessment of joint inflammatory activity in early rheumatoid arthritis: predictive value in disease activity and radiologic progression. Arthritis Rheum 2007;57:116-24.

61 Boesen M, Ellegaard K, Boesen L, et al. Ultrasound Doppler Score Correlates with OMERACT RAMRIS Bone Marrow Oedema and Synovitis Score in the Wrist Joint of Patients with Rheumatoid Arthritis. Ultraschall Med 2012;33:E166-72.

62 Haavardsholm EA, Ostergaard M, Ejbjerg BJ, et al. Introduction of a novel magnetic resonance imaging tenosynovitis score for rheumatoid arthritis: reliability in a multireader longitudinal study. Ann Rheum Dis 2007;66:1216-20.

\section{Answers}

1. A. T; B. T; C. T; D. F; E. F

2. A. T; B. F; C. T; D. T; E.T

3. A. T; B. T; C. T; D. T; E. F

4. A. F; B. T; C. F; D. F; E. F

5. A. T; B. T; C. T; D. T; E. T 